\title{
Religion and the novel
}

Guest-edited by

Dorothy Figueira 